\title{
Delorme like Transanal Haemorrhoidal Dearterialization for the Treatment of Haemorrhoidal Disease
}

\author{
Emanuel Cavazzoni , Emanuele Rosati, Luigina Graziosi , Elisabetta Marino and \\ Annibale Donini
}

University of Perugia School of Medicine, Departement of Surgery, Perrugia , Italy

Correspondence should be addressed to: Annibale Donini; dna@unipg.it

Received date: 9 Mars 2014; Accepted date: 16 October 2014; Published date: 16 March 2016

Academic Editor: Ioannis Kanellos

Copyright (C) 2016. Emanuel Cavazzoni , Emanuele Rosati, Luigina Graziosi , Elisabetta Marino and Annibale Donini . Distributed under Creative Commons CC-BY 4.0

\begin{abstract}
Transanal Haemorrhoidal Dearterialization (THD) is a new and effective technique for the treatment of haemorrhoids. The minimally invasive technique includes a step, called recto anal repair, when the rectal mucosa is lifted up with a series of sutures that once tied maintain an adequate mucopexy to treat the haemorrhoidal prolapse. In patients with a redundant prolapse the completion of the mucopexy can be challenging when the procedure is done according to the original technique description. The present modification is aimed to reduce the operative difficulties making the operation shorter, easier and effective.
\end{abstract}

Keywords: Haemorrhoidal, Dearterialization, Doppler, Technique

\section{Introduction}

Transanal Haemorrhoidal Dearterialization (THD) has been increasingly used as an alternative technique to conventional surgery for the treatment of haemorrhoidal disease (HD) [1]. The association of mucopexy to the simple dearterialization makes THD suitable for fourth-degree HD, when the prolapsing component plays a major role in symptoms development [2]. Performing THD, as originally described
[3], can be sometimes difficult in patients with a particularly redundant mucosal prolapse. Specifically, when the surgeon ties the first continuous suture to perform the mucopexy just below the dearterialization site, the adjacent rectal mucosa fold towards the dentate line creating a mucosal "pocket" which makes the next running suture difficult to be correctly placed (Fig. 1 a,b). The mucosal folding increases progressively with the number of completed sites of mucopexy,

Cite this Article as: Emanuel Cavazzoni , Emanuele Rosati, Luigina Graziosi , Elisabetta Marino and Annibale Donini (2016)," Delorme like Transanal Haemorrhoidal Dearterialization for the Treatment of Haemorrhoidal Disease", International Journal of Gastroenterology Research and Practice, Vol. 2016 (2016), Article ID 887550, DOI: 10.5171/2016.887550 
making the latest ones very difficult to identify and significantly shorter than the first (Fig. 2). Finally, the mucosal thickness increases progressively with the completion of the mucopexy, this leads to a more difficult identification of the underlying arteries by the Doppler probe which inevitably lies more distant from the vessels

(Fig.

3

a,b).

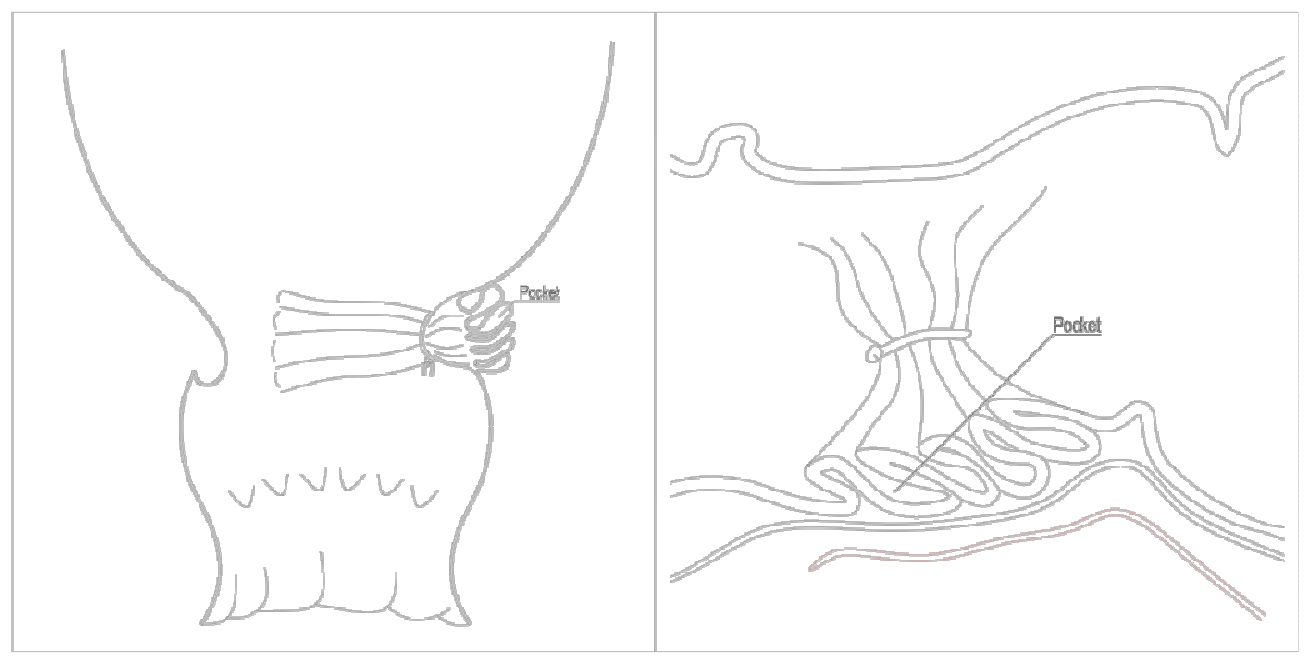

Figure 1 : a,b

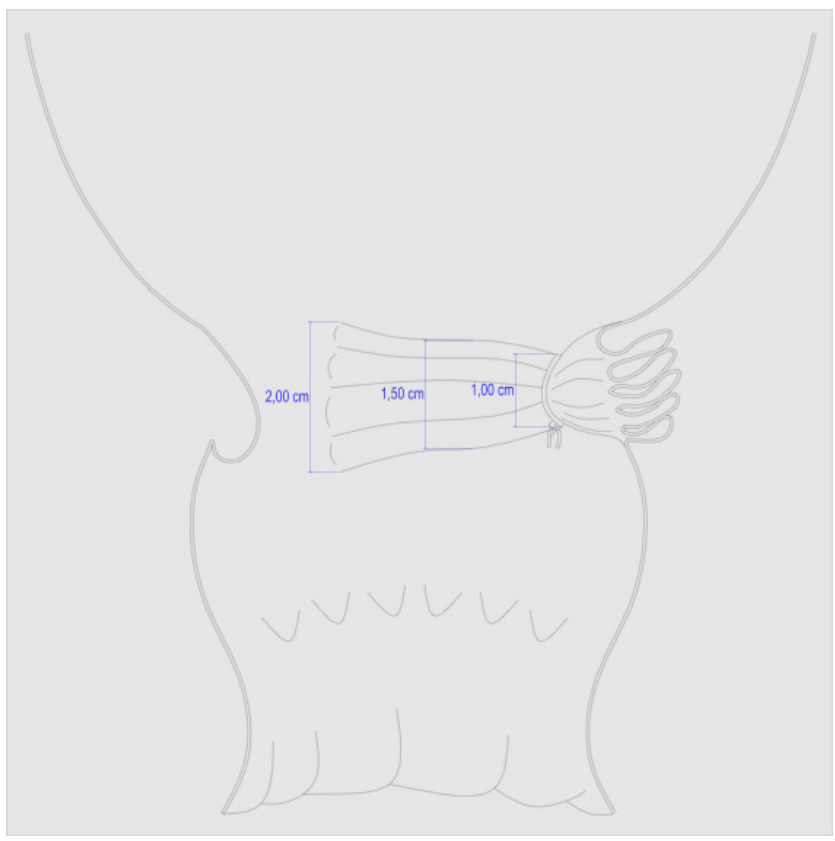

Figure 2

Emanuel Cavazzoni , Emanuele Rosati, Luigina Graziosi , Elisabetta Marino and Annibale Donini (2016), International Journal of Gastroenterology Research and Practice, DOI: 10.5171/2016.887550 


\section{Materials and Method}

The "Delorme Like" THD is a simple modification of the THD technique that differs from the original in the timing of the suture tying. The name derives from the similarity with the surgical procedure described by Delorme for the treatment of rectal prolaps. The equipment used is the THD system (THD S.p.A., Correggio, Italy) with the specifically designed proctoscope and the Doppler probe placed on the site of the device. The operation starts with the Doppler identification of the first branch of the superior rectal artery placed at 6-7 $\mathrm{cm}$ from the anal verge. We tent to place the first suture in correspondence of the rectal area with the largest prolapse, easily identified with a preoperative anoscopy. In males, this area usually corresponds with the posterior rectal wall (between 5 and 7 o'clock), while in women the anterior rectal aspect usually presents the most redundant mucosa (between 11 and 3 o'clock). The "Z" suture placed in correspondence of the arterial vessel is a transfixed stitch of 2-0 absorbable polyglycolic acid including the rectal mucosa and submucosa; the stitch is immediately tied and represents the cranial edge of the mucopexy. The same suture is then used to complete the mucopexy cranio-caudally making multiple mucosal-submucosal passages until the limit of about $1 \mathrm{~cm}$ from the dentate line is reached. Differently from the original technique, the mucopexy suture is not immediately tied, and the suture is secured to the surgical area with a small surgical clamp. Then, the next sites of dearterialization are identified and the same procedure is performed (Picture 1) until all the six major arterial branches are sutured (at 1,3,5,7,9 and 11 o'clock) and the running suture for the mucopexy are performed and left untied (Fig. 4; Picture 2). At this point, the anoscope is reinserted in the rectum and a Doppler double check is performed circumferentially to identify any residual vessel with a significant Doppler signal; those vessels are usually found at the even hours $(2,4,6,8,10,12)$ between the mucopexy sutures that are already placed. If a vessel is identified it is sutured with a single 2-0 absorbable polyglycolic acid "Z" stitch which is tied immediately. When the dearterialization is considered completed, the anoscope (without the sliding component that holds the Doppler probe) is reinserted in correspondence of the first site of mucopexy (Picture 3), and the running suture is then gently tied according to the original technique. The other running sutures are then tied in the same way and the operation is completed (Picture 4). 


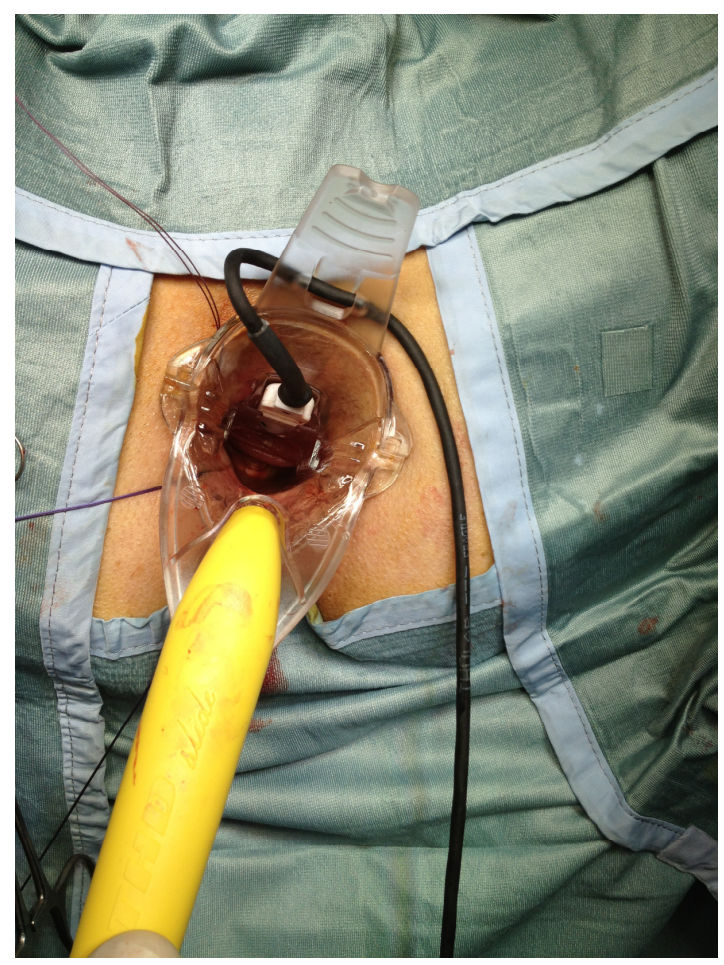

Picture 1

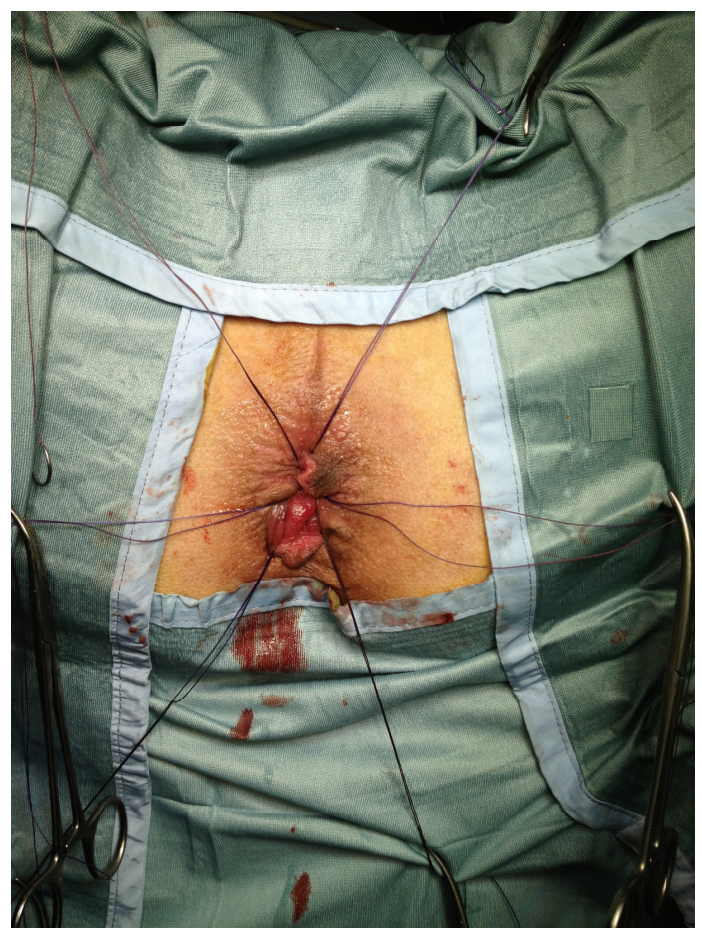

Picture 2

Emanuel Cavazzoni , Emanuele Rosati, Luigina Graziosi , Elisabetta Marino and Annibale Donini (2016), International Journal of Gastroenterology Research and Practice, DOI: 10.5171/2016.887550 


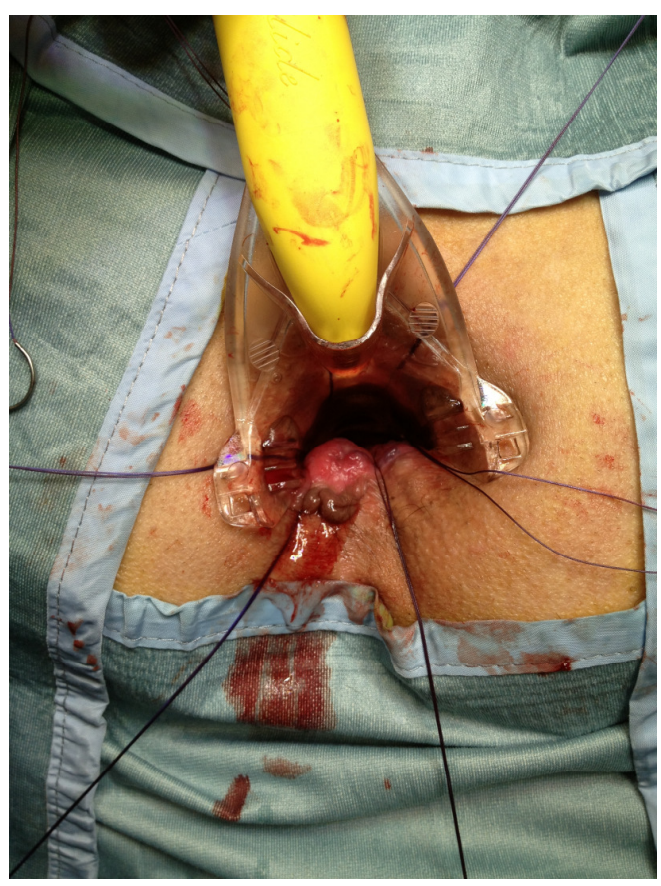

Picture 3

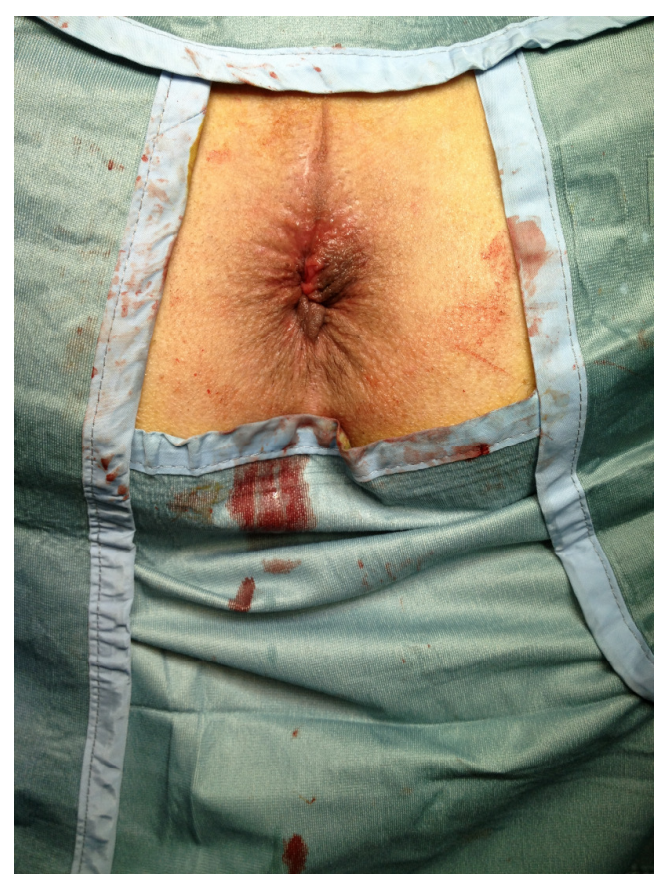

Picture 4

\section{Comparison with Other Methods}

THD is a valid technique to treat symptomatic HD. Even though, after more than one hundred procedures performed we often observed some difficulties in completing the mucopexy in correspondence of the last two/three sites, especially in patients with a severe rectal prolapse. The first mucopexy sutures 
significantly change the anatomy of the lower rectum, and the mucosa around the sutures crinkles and become less visible for the next mucopexy line; the mucosal folding reduces the length between the cranial dearterialization site and the dentate line, and usually the number of mucosal passages placed to complete the mucopexy decreases progressively from the beginning of the procedure to the last mucopexy site. The main obstacle in completing a correct and complete circular mucopexy is the creation of what we call mucosal pockets, originating by the craniocaudal folding of the higher part of the first mucopexy sites that literally hide large parts of the mucosa that are supposed to be included in the other suture lines (Fig. 5).

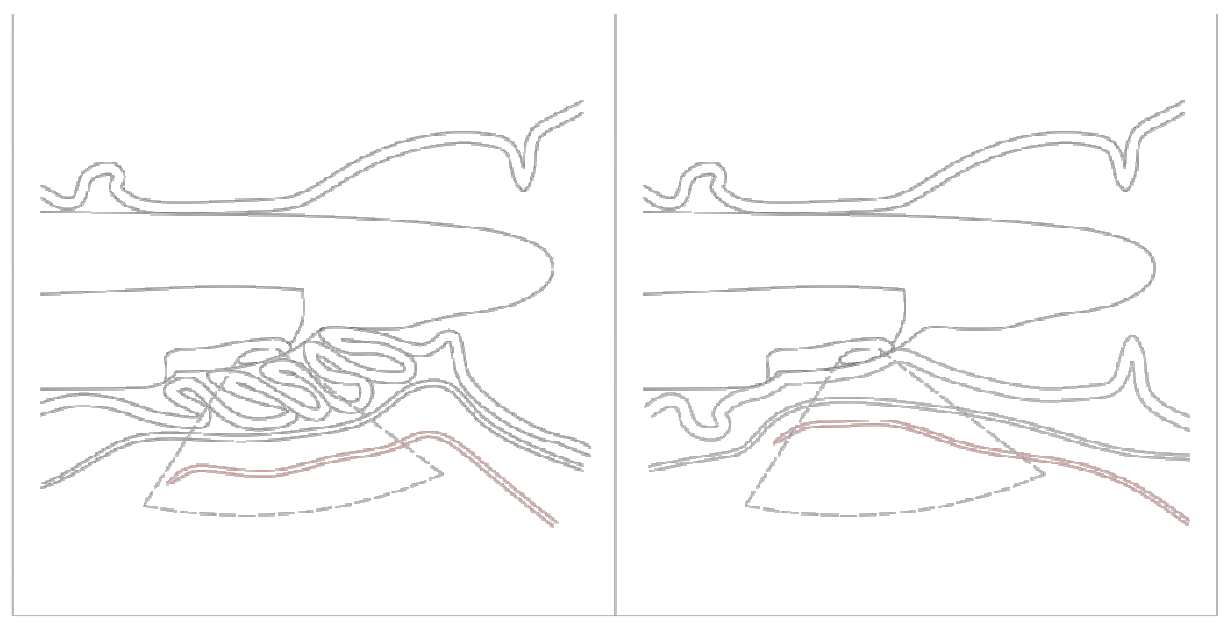

Figure 3 : a,b

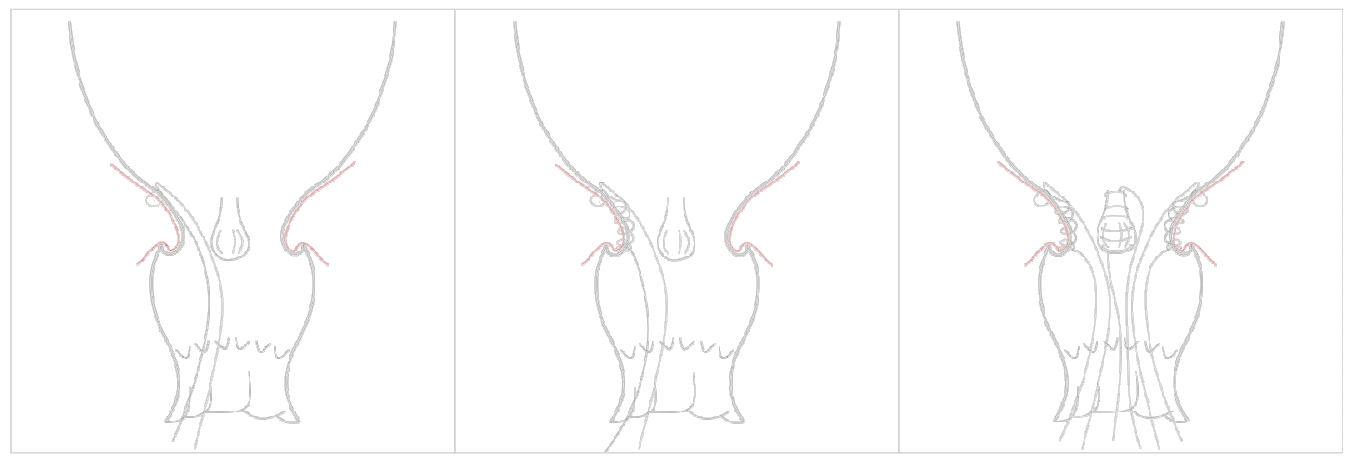

Figure 4

Emanuel Cavazzoni , Emanuele Rosati, Luigina Graziosi , Elisabetta Marino and Annibale Donini (2016), International Journal of Gastroenterology Research and Practice, DOI: 10.5171/2016.887550 


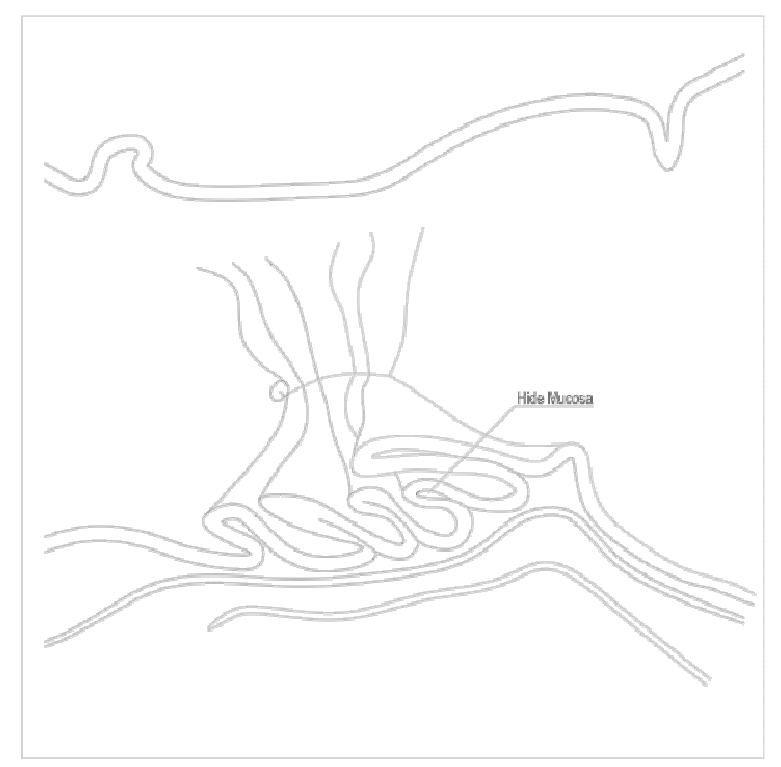

Figure 5

\section{Advantages and Disadvantages}

Advantages of the technique can be resumed as follows:

1) Avoiding the immediate tying of the running suture at several mucopexy sites allows a better identification of the major arterial branches, since the distance between the Doppler probe and the artery is represented only by the thickness of the rectal mucosa which is not folded in multiple layers (Fig. 3 a, b). This is true for the initial identification of the arteries at the main points of dearterialization $(1,3,5,7,9$ and 11 o'clock) as for any vessel residual to the major branch dearterialization that should be localised and sutured, if present.

2) The six mucosal-submucosal running sutures starting from the upper " $\mathrm{z}$ " stitch toward $1 \mathrm{~cm}$ from the dentate line are easier to place since there is no mucosal folding between the threads, resulting in a more straight mucopexy with no risk of over-suturing and with an easier identification of the rectal mucosa.

3) The avoidance of mucosal "pocket" between the sutures reduces the risk of rectal abscesses or areas of potential faecal impaction and retention [4, 5]. Furthermore, the rectal mucosa remains flat during the whole time of operation for every mucopexy site, increasing the visibility of the surgeon in placing the sutures.

4) Avoiding the mucosal overlapping between adjacent sutures can reduce the post-operative oedema and mucosal swelling that we believe can be responsible of post-operative pain and tenesmus.

\section{Difficulties and Complications}

In our opinion, the only possible adjunctive difficulty in comparison with the original technique could be the intra operative bleeding originating from the several running sutures that are left untied until the end of the procedure. In our experience, though, we observed that the continuous use of the proctoscope during the operation offers a valid compression on the suture sites that effectively controls the bleeding while the mucopexy sticthes are left untied. In our personal experience, the adoption of the technical modification resulted in an overall reduction of the operating time (about 30 minutes to complete the whole procedure compared to 40-45 minutes of the original technique), 
which indirectly confirms that the suture placement is easier and the modification does not add any significant intra operative difficulty. In our series of more than 20 patients (12 M; $8 \mathrm{~F}$ ) with a severe prolapse associated with haemorrhoids treated with the modified technique we experienced a mild reduction of post operative pain measured by a 1-10 visual scale at 24,48 and 72 hours from operation (mean values $3.5 ; 3.35 ; 2.55$ respectively), no differences in hospital length or readmission and a reduction of early (within 7 days) and late (within 30 days) tenesmus and anal discomfort when compared with our historical cohort of about 120 patients. Further studies comparing the modified technique with the original one might demonstrate some advantage in terms of post-operative pain, morbidity and efficacy.

\section{References}

1. Infantino A. Transanal haemorrhoidal artery echodoppler ligation and anopexy (THD) is effective for II and III degree haemorrhoids. A prospective multicentre study. Colorectal Dis. 2010 Dec;12(12):1274.

2. Ratto C, Giordano P, Donisi L, Parello A, Litta F, Doglietto GB. Transanal haemorrhoidal dearterialization (THD) for selected fourth-degree haemorrhoids. Tech Coloproctol. 2011 Jun;15(2):191-7

3. Gupta PJ. Transanal haemorrhoidal dearterialization. Tech Coloproctol. 2008 Jun;12(2):138-40

4. Basso L, Pescatori M, La Torre F, Destefano I, Pulvirenti D'Urso A, Infantino A, Amato A; AECO Group. Emerging technologies in coloproctology: results of the Italian Society of Colorectal Surgery Logbook of Adverse Events. Tech Coloproctol. 2013 Apr;17(2):207-11

5. Berkel AE, Witte ME, Koop R, Hendrix MG, Klaase JM. Brain abscess after transanal hemorrhoidal dearterialization: a case report. Case Rep Gastroenterol. 2013 May

18;7(2):208-13. 